S sciendo

The Impersonal 'you' and Other Indexicals

Stefano Predelli

University of Nottingham

Disputatio No. 8

May 2004

DOI: $10.2478 /$ disp-2004-0001

ISSN: 0873-626X 
Disputatio 16, May 2004

\title{
The impersonal 'you' and other indexicals
}

\author{
Stefano Predelli \\ University of Nottingham
}

\begin{abstract}
In this essay I propose a semantic analysis of impersonal uses of 'you', and related uses of other indexical expressions. The framework I employ is Kaplan's classic analysis of indexical languages, enriched with independently motivated hypotheses about the identification of the semantically relevant context, and about the employment of generic expressions.
\end{abstract}

Consider an utterance of

(1) you get in trouble with that move

said by a chess instructor to a student. More often than not, such an utterance is to be understood impersonally, in the sense that, roughly, chess players typically get in trouble. After all, (1) may well occur within a true fragment such as

$\left(1^{*}\right)$ according to all textbooks, you get in trouble with that move

even though textbooks obviously do not contain warnings directed to the particular student the instructor is addressing (see Nunberg 1993, 21). Similar, straightforward instances of the impersonal use of 'you' are provided by cases such as 'you just can't tell' or 'you can lead a horse to water but you can't make it drink', which convey general information, rather than a content pertaining to the addressee's inabilities.

Although the impersonal behavior of 'you' has not received a great deal of attention, its analysis plays a non-peripheral role with respect to certain fundamental issues in contemporary philosophy of language. One 
among the most influential currents in philosophical semantics, the tradition within so-called 'direct reference theory', has found important argumentative support in the analysis of indexical expressions, in particular simple indexicals such as 'I' and 'you'. In this tradition, although these expressions are indeed endowed of a descriptive conventional meaning (character), this descriptive condition does not contribute to the semantic content they encode. 'So, in this view, my utterance of 'I am hungry now' is associated with the singular content pertaining to my appetite, and not with any descriptive, general proposition — in particular, not with the proposition that the speaker is hungry at the time of utterance. Yet, the intuitive behavior of 'you' when occurring in typical utterances of (1) seems to resist an interpretation along these lines: the content conveyed is the apparently general information that any individual generally gets in trouble with a certain move. One prima facie obvious reply to this worry is that 'you' is lexically ambiguous, and that, although it may occasionally behave in ways incompatible with the standard theory, in a variety of straightforward instances it instantiates the pattern dear to the singularproposition theorist. The problem with this suggestion is that, as I explain in the first paragraphs of section one, such a lexical ambiguity claim is independently suspicious. Its rejection, and the consequent search for a unified account, may therefore point towards more radical directions, such as the notion that 'you' does not at all fit the semantic pattern described in the classic accounts of indexicality. I critically discuss a suggestion in this spirit in the remainder of section one, where I focus on Geoffrey Nunberg's analysis of indexicality.

The main aim of this essay is that of providing a semantic analysis of cases such as (1). As I argue in sections two and three, the intuitively required outcome may be obtained by appealing solely to independently motivated results, such as considerations pertaining to the choice of the semantically relevant contexts, and a relatively straightforward treatment of generic sentences. The picture resulting from this approach turns out to be straightforwardly compatible with the traditional account of the meaning of indexicals. In particular, the possibility that examples such as (1) convey information of a general import does not contradict the notion that, in their

${ }^{1}$ See in particular the classic accounts in Kaplan 1977 and Lewis 1980. 
more customary uses, indexical expressions non-ambiguously contribute to the expression of singular contents.

\section{Ambiguity and generality}

According to the classical account, indexicals may be interpreted only with respect to a context. In the formal literature, contexts are understood as $n$-tuples containing the appropriate parameters. Thus, for instance, a context for a language containing 'I', 'you', and 'now' may be interpreted as an $n$-tuple $<_{c_{i}}, c_{a}, c_{t}>$, where $c_{i}$ and $c_{a}$ are individuals (respectively the agent and the addressee of the context) and $c_{t}$ is a time. The (semantically relevant portion of the) meaning of the aforementioned indexicals may then be understood as a function from contexts to semantic values, that is, as what is customarily called a character. For example, the character for ' $I$ ' is presumably the function which, given a context $c$, yields the agent of $c$ as that expression's referent; similarly, the character for (the singular) 'you' is apparently the function which, when applied to $c$, yields $c_{a}$, the context's addressee.

It is a consequence of the lexical ambiguity analysis of (1) that 'you' is associated with two distinct characters. In one case, what is allegedly at issue is the aforementioned function rendering the addressee; in the other case, the appropriate character is presumably a constant function responsible for the generation, in any context, of the content that anyone gets in trouble with a certain move. Characters are intended as formal representations of the semantic behavior conventionally associated with a given expression by the regularities of the language. It follows that, according to the hypothesis of lexical ambiguity, the use of 'you' for either singular or impersonal purposes is entirely fortuitous: it is a mere accident of the conventions governing the English language that expressions with the same spelling and pronunciations happen to be employed in these ways. ${ }^{2}$ This is an important aspect of the lexical ambiguity approach, which, as I explain in the next paragraphs, is ultimately responsible for its inability to provide a satisfactory account of impersonal uses of 'you'. Note in par-

${ }^{2}$ For a more sophisticated version of the ambiguity thesis, see Smith 1989: in Smith's view, indexicals are associated with a unique metacharacter, which in turn yields a variety of distinct characters in different settings. 
ticular that, according to this view, what is at issue is not merely the notion that 'you' may be used ambiguously, typically as a pronoun referring to the addressee, but occasionally as contributing to the presentation of general information. That 'you' is ambiguous in this sense is an indisputable datum. This harmless consideration is however not by itself an explanation of the phenomenon under study here, but merely a description of the explanandum: what is needed is an account of why such a duality of uses is available. The hypothesis of lexical ambiguity is an attempt at providing a reply to this question. In this view, the dual use of 'you' is reducible to the presumed accidental fact that, in the historical development of the English language, two distinct meanings have been associated to the lexical item in question.

The ambiguity thesis discussed in this section is thus an explanation grounded on a claim of accidental ambiguity. Claims of this kind are typically contrasted with proposals of systematic ambiguity. As an example of ambiguity of this latter type, consider for instance so-called sententialist analyses of expressions occurring in embedded clauses within attitude reports. At least in some of these views, simplifying considerably, a name such as 'Cicero' contributes its referent when occurring in simple sentences, but supplies a richer item, say, the pair consisting of Cicero and the name 'Cicero', when occurring within a that-clause. ${ }^{3}$ The discussion of the merits and disadvantages of such analysis of proper names and belief reports is not the topic of this essay. However, this proposal provides a pedagogically instructive example of the consequences of an approach in terms of systematic ambiguity: the presumed dual behavior of 'Cicero' is not explained by attributing two meanings to this name, but rather by postulating a particular semantic phenomenon, regarding the interaction between proper names and attitude predicates or complementizers. It follows that, in this view, a parallel dual behavior is attributable to all proper names, not just to 'Cicero', and is furthermore to be expected for occurrences of belief reports and proper names in languages other than English: any language containing names and constructions parallel to the English 'believes that' will presumably involve the cases of reference-shifts the theory envisions for 'Cicero'.

${ }^{3}$ See for instance Richard 1990. 
Returning to the hypothesis under discussion in this section, its rejection of an analysis in terms of systematic phenomena entails that conclusions similar to those in the foregoing paragraph are not to be expected. In the lexical ambiguity view, the dual behavior of 'you' is reducible to the accidental fact that one expression ends up being associated, by virtue of the conventions regulating English, with two characters, just as is the case with, say, 'bank' or 'bill'. It would thus be surprising if an importantly parallel duality of use were available for indexicals other than 'you', and if such duality were present in the translation of 'you' in at least some other languages - just as it would be highly surprising if most English expressions such as 'bank' were similarly ambiguous, and if an analogous ambiguity were to be found in the translation of 'bank' into other, unrelated languages. Yet, as I explain in the following paragraphs, the case of the impersonal use of 'you' is different from that of straightforward instances of lexical ambiguity precisely in these two respects.

To begin with, the two-fold behavior exemplified by (1) is reflected by an analogous duality in the semantic profile of translations of (1) in a variety of other languages. To cite just a few, sufficiently unrelated Indo-European languages, the use of the singular, second-person pronoun in an impersonal sense is common in German, Italian, Dutch, French, and the Scandinavian languages. Here are the translations of (1) into Italian and Dutch, were the italicized expression, typically employed to designate the addressee, is instead used in the impersonal sense:

(1**) elk handboek zegt dat je in moeilijkheden komt

$(1 * * *)$ secondo tutti i manuali, ti trovi in difficolta' con questa mossa. ${ }^{4}$

${ }^{4}$ As for Portuguese, Marco Ruffino suggested to me 'Você nunca sabe se um político está falando a verdade' as an instance of the impersonal 'you'. Many thanks to Francois Recanati, Filip Buekens, Max Kölbel, and Marco Ruffino for their input as native speakers. The evidence provided by, among others, German or Italian, is furthermore particularly significative, given that these languages are sensitive to the distinction between familiar and formal second-person pronoun ('du' vs. 'Sie', or 'tu' vs. 'lei'), a distinction uncontroversially encoded in the lexical meaning conventionally associated with these expressions. Suppose for instance that the Italian expression referring to the addressee, namely 'tu', were only accidentally homophonic with the expression used for impersonal purposes. In this case, it would be highly surprising if part of the former's meaning, that 
It should be stressed that the evidence under discussion does not amount to the untenable claim that all natural languages display the duality under discussion. Indeed, it will become an important question for analyses in terms of systematic mechanisms that the regularities they propose do not overgenerate, and that they be compatible with the fact that, in a sense to be explained, the impersonal use of 'you' is sanctioned by conventional features of one type or another — on this issue, see the discussion on 'limited productivity' in the concluding paragraphs of section three. What matters at this stage is however the weaker indication, sufficient to cast at least initial doubt on the lexical ambiguity hypothesis, that some languages other than English display a dual use for expressions translating 'you'.

Further support for these considerations is offered by English examples that involve indexicals other than 'you', and which apparently instantiate an importantly similar phenomenon. Consider

(2) do not put off until tomorrow what you can do today.

This sentence involves temporal indexical expressions such as 'today' and 'tomorrow', namely expressions typically employed so as to refer, respectively, to the day of utterance and to the day following it. Still, at least more often than not, (2) is not employed in order to convey the information that a particular day is unsuitable for procrastination, but rather to impart the more general notion that, for any given day, what can be done at that time should not be postponed. This reading is confirmed not only by the intuitions of normal English speakers, but also by the more typical example of

$\left(2^{*}\right)$ never put off until tomorrow what you can do today.

This sentence involves a temporal operator 'never', which would be inexplicable on the assumption that 'tomorrow' and 'today', occurring

pertaining to its familiar character, were inherited by the latter. Yet, this is precisely the case: $(1 * * *)$, though unquestionably not referring in particular to the addressee, is unsuitable for a conversation with someone deserving of the formal form of address. (Thank you to Buekens and Kölbel for calling my attention to phenomena of formality). 
within its scope, behave in their more typical, referring guise. As is well known, were 'today' and 'tomorrow' contributing the day of utterance and the day following it, they would remain insensitive to the effects of intensional operators: 'never' would remain unaccountably vacuous.

Given the analogies between the behavior of 'today' in such utterances of (2) and that of 'you' in (1), it seems plausible to conclude that systematic semantic regularities are at work, responsible for an indexical's ability to occasionally partake in the encoding of non-singular propositions. The case of (2) is particularly telling against explanations in terms of lexical ambiguity because it involves two temporal indexicals, whose interpretation must apparently be carried out in a uniform manner. If each of the expressions 'today' and 'tomorrow' where lexically endowed of a double character, (2) ought to be interpretable in four different manners. Thus, for instance, the presence of the two lexically ambiguous items 'duck' and 'bank' in 'I saw her duck by the bank' generates four readings. Yet, such a four-fold ambiguity does not seem to be available for (2), which may only be interpreted either in an unusual specific manner, where 'today' and 'tomorrow' are both anchored to the day of utterance, and in its more customary non-specific connotation.

Incidentally, it is instructive to note that the case of (2) is also reproducible in the languages alluded to above. German has 'verschiebe nie auf morgen, was du heute kannst besorgen'; in Dutch, 'doe nooit morgen wat je vandaagal kan doen', and in Italian 'mai rimandare a domani quello che $t u$ puoi fare oggi'. Even more significative is the fact that parallel examples may be found, in English as in other languages, involving other temporal indexicals, such as 'now' in typical uses of 'better keep now than seek anon', location indexicals, as in 'home is where the heart is', and even the first person indexical 'I' (see later for cases of this kind). ${ }^{5}$ Once again, it should be noted that the regularity apparently at work in all these examples does not inevitably extend to all instances of indexicality, and displays

${ }^{5}$ In this respect, it is also interesting to note the pattern instantiated by examples that are commonly classified as purely idiomatic, such as 'he smokes now and then', 'this is neither here nor there', 'Kermit was jumping here and there', and 'I did this and that'. Another (negotiable) case is provided by 'they', a pronoun customarily employed to denote a class of contextually demonstrated individuals, but used impersonally in 'they say it is going to rain', at least when understood as 'it is said that it is going to rain'. 
the type of 'limited productivity' that shall be addressed later in this essay: it is apparently impossible to utter, say, 'you are always hungry now' in order to convey that one is hungry at any time. But although constraints on the systematic mechanisms governing cases such as (1) and (2) appear to be operative, what suffices for the purpose of this section is the notion that some interestingly distinct examples apparently display the pattern characteristic of 'you'. This much, as I explained above, is at odds with the postulation of lexical ambiguity, and points towards an explanation in terms of systematic semantic regularities.

The conclusion that lexical ambiguity does not provide a satisfactory explanation of (1) may indirectly be interpreted as evidence that the semantic behavior of 'you' (and 'today' and 'tomorrow'), though not explainable in terms of a multiplicity of characters, is in fact more complex than usually assumed. According to the traditional approach, the conventional meaning assigned to 'you' is the function rendering the context's addressee. Yet, so it may be objected, although this hypothesis renders the correct analysis for certain uses of (1) or (2), it seems clearly unable to account for the non-specific readings of these sentences: in the classical view, only a singular proposition pertaining to a certain individual or day may semantically be associated with these sentences. On the basis of such reasoning, Nunberg has suggested that the classical account, though inspired by certain undeniable aspects of the behavior of indexicals, fails to provide an adequate picture (see Nunberg 1993). ${ }^{6}$ What the problematic readings of (1) or (2) allegedly indicate is the need for a theory able to explain an indexical's ability to contribute general propositions, such as the proposition that one gets in trouble with a certain move. In Nunberg's theory, then, although an expression such as 'you' is unquestionably related to the addressee of the context, the selection of such individual does not exhaust its semantic function: in virtue of a variety of background considerations, a particular 'relational component' may be employed so as to determine, on the basis of the selected individual, a suitable property. In straightforward situations, so Nunberg argues, the relational component is, trivially, the identity relation. But in other cases, the relational component may intervene at a level prior to the establishment of semantic content, and lead from the individual in ques-

${ }^{6}$ For a discussion of Nunberg's analysis, see also Recanati 1993. 
tion to a salient property that individual instantiates. One of Nunberg's favorite examples involves the first person indexical 'I' (Nunberg 1993, 20):

Condemned prisoner: I am traditionally allowed to order whatever I like for my last meal.

In Nunberg's view, the conventional profile of 'I' initially selects the prisoner, but the semantic content conveyed by the utterance in question is not the singular content pertaining to that individual. On the basis of his salient properties, in particular the property of being a death row inmate, the appropriate relational component allegedly determines the appropriate semantic contribution, thereby generating the general content that, roughly, condemned prisoners are traditionally allowed whatever they like for their last meal.

It is an important advantage of Nunberg's theory that it seeks for a systematic explanation of the phenomenon under study: presumably, the contextually suitable relational component maps all indexicals to an appropriate semantic value, thereby accounting for the regularities inexplicable from the point of view of the lexical ambiguity account. On the other hand, Nunberg's solution does not suffice as a presentation of the systematic connection under discussion, insofar as it does not provide a complete analysis of the desired semantic profile. Even if a property were identified by a 'relational component', what remains to be explained is how it comes to participate in the truth-conditional behavior for, say, the prisoner's utterance. Clearly, it is not the property of being a death-row inmate that is generally allowed a last meal; what is thus required is a systematic account of how, on the basis of such property, one manages to reach the result that typical instances of it are free to choose their final repast. More importantly, the suggestion that a certain property is individuated on the basis of the individual selected by the indexical, though prima facie adequate for the prisoner's example, does not seem to be generally correct. As an explanation of the customs on death-row, I may tell you

you are traditionally allowed to order whatever you like for your last meal,

in the impersonal sense that prisoners may choose their last meal. However, in this case, no plausible relational component may derive, from the 
individual selected by 'you', namely yourself, the property of being a death row prisoner (at least not in any non ad hoc, explanatorily interesting manner). Similar considerations hold with respect to Nunberg's own example of the impersonal 'you', with which I have begun this essay:

Chess teacher giving an introductory lesson to a student who has just played 4. NxP ...: According to all the textbooks, you often get in trouble with that move.

In Nunberg's interpretation, this amounts to the claim that ' $\ldots$ the person who plays 4 . NxP often gets in trouble', namely a claim involving a property (being someone who plays $4 . \mathrm{NxP}$ ) instantiated by the addressee. Yet, although a reading of this type may occasionally be intended by the speaker, a more appropriate gloss would rather suggest that

generally, chess players of normal abilities get in trouble with that move.

The teacher's utterance is therefore intuitively appropriate even if it were addressed to an exceptional player who, unlike most people, always succeeds in employing 4 . $N_{x} P$ as part of a winning strategy:

Chess teacher to a master who has just played 4 . $N_{X} P$...: According to all the textbooks, you often get in trouble with that move. But of course you may be able to get away with it.

In what follows, I suggest an alternative analysis of cases such as (1) or (2), which avoids the shortcomings of the hypotheses briefly discussed thus far. As a preliminary, I begin by discussing certain independently motivated considerations pertaining to the use of indexicals. I thus temporarily abandon the study of the impersonal use of 'you' in the initial paragraphs of the following section. I return to my main topic at the end of section two, where I present the first part of my proposal.

\section{Context of utterance and context of interpretation}

In typical instances, the semantic interpretation of an utterance reaches the desired results by evaluating the indexicals within the uttered sentence with respect to the context of utterance. For instance, according to the character for 'I', this expression refers to the individual that plays 
the role of agent in the context; more often than not, such a role is instantiated by the speaker, namely by the person who is producing the utterance under analysis. Similarly, in typical scenarios, 'now' refers to the temporal parameter in the context of utterance, namely, to the time when it is being uttered. The notions that 'I' refers to the utterer and that 'now' refers to the time of utterance are therefore adequate rules of thumb, responsible for the correct conclusions in many straightforward cases. Not always, however: in some interesting instances the intuitively correct results are obtained by evaluating the uttered sentence with respect to contextual parameters different from those provided by the context of utterance. Consider the following example. In 2002, during a vivid narration of certain events of World War II, I say:

The allied troops cannot wait any longer. The time has now arrived for the invasion of Normandy. ${ }^{7}$

My utterance does not convey the patently false proposition that 2002 is the appropriate time for a military invasion of Northern France. But it also counterintuitive to suppose that such a semantic effect is achieved in virtue of an ad hoc character for 'now', alternative to its customary ability to select the contextually appropriate time. Note in particular that a perfectly parallel behavior is displayed by the use of the present tense. Hence, it is plausible to suppose that the verbal tenses, 'now', and any other temporal indexical I may have chosen, proceed with their customary semantic profile, interested in the context's temporal co-ordinate, but are evaluated with respect to a context distinct from that supplying the time of utterance. This suggestion fits rather nicely with the vague but correct intuition that the speaker is 'putting himself in the shoes' of an observer reporting the events the day before D-day, and that such a rhetorical device is responsible for a certain effect of vividness. In what follows, I refer to the context relevant for the semantic interpretation of an utterance as the context of interpretation. It is a consequence of the approach sketched thus far that, in some cases, the context of interpretation does not coincide with the context of utterance: for instance, the temporal co-ordinate appropriate for the interpretation of 'now' or the

${ }^{7}$ This example and the next are borrowed from Predelli 1998. 
present tense may differ from the time of speaking. Similar considerations hold for other indexicals, for instance 'here'. Consider the following passage:

here, to the sheltered columned coolness, Ramanujan would come. Here, away from the family, protected from the high hot sun outside he would sometimes fall asleep .... (Kanigel 1991, 29-30).

The correct interpretation of this passage does not depend upon the identification of the location where the text was written: the spatial indexical 'here' is rather anchored to a context distinct from the context of utterance, and presumably including a location intended as salient by the author.

In this view, then, indexical expressions such as 'now' or 'here' are associated with a unique character, namely the functions that, given a context $c$, render respectively the time and location of $c$ as semantic value. When such characters are applied to the analysis of particular utterances, the context that often needs to be taken into consideration is the context of utterance: in these straightforward cases, the characters in question end up selecting the time and place of utterance. The examples I presented in the foregoing paragraphs indicate that such situation, albeit typical, is not inevitable: on suitable occasions, the context of interpretation may fail to contain the co-ordinates of utterance. What exactly is responsible for the selection of a particular context as semantically relevant is a difficult issue. The speaker's intentions may play a role in this respect, although, as I suggest in what follows, they may perhaps be accompanied by other factors. Still, although these tentative hypotheses obviously do not suffice as a complete theory for the phenomenon in question, the choice of one explanation rather than another is not a peculiar burden of the view put forth thus far. In the classic semantic approach to indexical languages, what is evaluated are pairs consisting of a particular syntactic construct and a context, in turn supposed to represent a particular utterance. In the foregoing paragraphs, I focused on the question of the context appropriate for the representation of an utterance, and I hinted that it may differ from the context of utterance. Yet, independently from the peculiarities of indexicals, and in pretty much anybody's understanding of the structure of semantic evaluation, nontrivial decisions need also to be taken with respect to the syntactic con- 
struct appropriate on a given occasion. If I say 'every person speaks a language' is my utterance appropriately interpreted as saying that there is a language every person speaks? The answer to this question requires a decision pertaining to the syntactic construct suitable for my utterance, that is, a choice between, roughly

(for every person $\mathrm{x}$ ) (there is a language $\mathrm{y}$ ) (x speaks $\mathrm{y}$ )

and

(there is a language $\mathrm{x}$ ) (for every person $\mathrm{y}$ ) (y speaks $\mathrm{x}$ ).

How are we to decide? My intentions, my audience's expectations, the topic of the conversation, may all provide interesting clues. Any hypothesis one may wish to explore in this respect ought to be immediately applicable to the question pertaining to the identification of the semantically relevant context, whenever it does not coincide with the context of utterance. $^{8}$

A related worry needs to be addressed before I continue. That, for instance, 'I' or 'now' may be employed so as to select parameters that do not figure in the context of utterance does not entail that one may employ these expressions at will, so as to refer to any individual or time she may wish. It is simply not possible for me to utter, out of the blue, 'I am now the President of the United States' in order to convey that Bill Clinton was the American President in 1995, no matter how hard I intend to refer to that man and that year. This conclusion is however not only compatible with the approach I suggested, but it is in fact easily explained by it. There are obvious pragmatic motivations for the undeniable supremacy of the parameters in the context of utterance in normal situations, such as the fact that, in the vast majority of cases, a certain instant or period is suitable for the role of the contextually salient time in so far at it is the time when the utterance under evaluation is being produced. For this reason, strong motivations are required in order to raise to salience a context distinct from the context of utterance, and the interpretive effort involved in the analysis of cases of this type is consequently

${ }^{8}$ For a discussion of some related questions, see for instance Corazza et al. 2002 and Romdenh-Romluc 2002. 
non inconsiderable. Any competent user of English is expected to understand my utterance of, say 'now the troops are ready for the invasion' as referring to the contextually salient time. Yet, at least as long as she is unfamiliar with the rhetorical custom involved in historical narrative, she may well fail to understand my remark as referring to a relevant time in the past, rather than to the time of speaking. A sufficiently explicit set of background information may be not only helpful, but essential if successful communication is to take place: I may, for instance, begin my narrative with explicit announcements such as 'it is now 1945 ...'.

Particularly relevant in this respect is also the presence of relatively well established practices, such as the employment of the present tense to refer to a time in the past. It is the existence of communicative practices of this type that makes it the case that some instances of the contextual shifts envisioned here are more often employed than others, and have thus become relatively standardized: it is not uncommon, for instance, to list the ability to refer to a salient past time as part of the entry for 'now' in common dictionaries. For the purpose of this essay, it suffices to note that such indications should not be interpreted along the lines of the lexical ambiguity approach: as far as its semantic behavior goes, 'now' is associated with one unique character, namely the function yielding the contextually appropriate time. How a certain period may be raised to conversational salience is a distinct question, related to the possibility of using such an expression in order to communicate a content pertaining to a particular time: conventionalized settings, such as the practice of historical narrative, facilitate this type of communicative aim, and thus provide run of the mill instances of the phenomenon under discussion.

To an important extent, the distinction between context of utterance and context of interpretation is relevant also for the examples with which this essay is concerned, such as (1) or (2). On the assumption that, for instance, the character for the singular personal pronoun 'you' is the function yielding the context's addressee, it must be the case that the context appropriate for the interpretation of the instructor's utterance of (1) is not the context of utterance: the content she intuitively conveys is not the singular proposition that the person she is talking to gets in trouble with that move. Similarly, given a typical utterance of (2), it must be the case that its context of interpretation does not contain the coordinates of utterance: (2) does not generally convey a singular content pertaining to the day of speaking. On the other hand, a further important 
property characterizes the examples under discussion: it is not only the case that the addressee is unsuitable as a referent for 'you' in (1), but it is also the case the no other particular individual would do in this respect. By the same token, it is not only true that (2) does not refer to the day of speaking and the day following it: its correct interpretation does not pertain to any particular pair of dates at all, and rather conveys a message of a general import. So, if the hints in the foregoing paragraphs are extended to these cases, the appropriate contexts of interpretation not only fail to provide the co-ordinates of utterance, but fail to supply any parameter at all to the interpretation of some expressions: no particular individual plays the role of addressee in the context for the interpretation of (1), and no particular time occupies the temporal co-ordinate in the context for the interpretation of (2).

I refer to contexts of this type as gappy contexts. Formally, the notion may be defined along the following lines. Let a context appropriate for the interpretation of a fragment containing, say, 'I', 'you', and some temporal indexicals, be a triple $\left\langle C_{i}, C_{a}, C_{t}\right\rangle$, where $C_{i}, C_{a}$, and $C_{t}$ are sets, and are such that they are either the empty set or a singleton containing, respectively, an individual (the agent of the context), another individual (the addressee), and a time. The character for, say, 'I', may then be represented as the function which, given a context $c$, returns the unique element of $C_{i}$ if $C_{i}$ is non-empty, and does not provide any semantic value if $C_{i}$ is the empty set (similarly, mutatis mutandis, for the other indexicals). Incidentally, as an example in which a context of interpretation gappy with respect to the agent co-ordinate seems appropriate, consider a discourse such as

So Jane wants a puppy and they buy her this nice terrier. Now, if I have a dog, I should take good care of it, right? Well, now that she's got one, Jane seems to be entirely uninterested in that poor thing.

The sentence 'if I have a dog, I should take good care of it' as it occurs in this fragment does not provide irrelevant information regarding my duties towards animals, and does not encode a content pertaining to any particular individual at all: rather, it imparts the notion that, typically, someone who has a dog ought to take good care of it.

The distinction between context of utterance and context of interpretation, and the notion of a gappy context of interpretation, provide 
important first steps in the analysis of the uses of indexicals under discussion. But these conceptual tools are obviously not sufficient: the analysis proposed thus far correctly eliminates the wrong interpretation, one referring to particular individuals such as the speaker or the addressee, but is unable to provide the intuitively correct reading. All we are left with at this stage are seemingly non-evaluable instances, in which certain indexicals are entirely deprived of semantic value. Yet, closer analysis of the intuitive behavior of sentences such as (1) or (2) provides tentative indications of a promising direction: for instance, as I already remarked in my discussion of Nunberg's proposal, impersonal uses of (1) convey the information that typically players get in trouble with a certain move. An interpretation of this type seems intriguingly similar to a particular reading of other sentences involving certain denoting expressions; for instance, the sentence 'a wolf takes a mate for life' is often employed generically, as indicating that typical, normal wolves tend to take a mate for life. It is thus fitting that I briefly pause on cases of this kind, before I explain how, on the basis of independently motivated considerations, the hints I provided thus far may be developed into a theory of the nonspecific uses of indexicals.

\section{From generics to indexicals}

What is important from the point of view of the strategy to which I alluded is not the development of a full-fledged theory of genericity, or the endorsement of a particular approach to generics. Still, for the sake of presentation, it is desirable that I present my considerations within a fairly well established semantic framework, one able to account for both generics and indexicals. In this section, I opt for Discourse Representation Theory (DRT), but I remain deliberately neutral with respect to a variety of non-immediately relevant and possibly controversial details in alternative versions of this semantic approach. As a result, most of my conclusions ought to be easily rephraseable within other theoretical environments sufficiently powerful for dealing with the phenomena under discussion.

Before confronting generic sentences, I begin with a short introduction to the very general traits of DRT. In the apparatus of DRT, a sentence such as, say, 'a man is running' is associated with a structure roughly along the lines of 
[ $x$ is a man] [ $x$ is running],

which is in turn interpreted as true iff there is a value for $x$ that verifies the conditions in question, i.e., iff $\exists_{x}(x$ is a man $\& x$ is running $) .{ }^{9}$ Incidentally, independently of the details in DRT and of the peculiarities of the problems discussed in this essay, the choice of the appropriate evaluations of the variable is typically contextually constrained. Suppose for instance that, after you complained that the local park is deserted, I point at a distant individual and say 'a man is running'. Intuitively, my utterance is true iff there is a salient individual who is a man and is running: if nobody was in the park, my utterance would not have been verified by the fact that, somewhere in a distant country, some man was indeed running. This well-known issue, related to so-called 'domain restrictions', is straightforwardly reflected within the apparatus I am sketching, in terms of contextual constraints on the class of available values for the variable: an utterance of 'a man is running' turns out true iff there is a (contextually suitable) value for $x$ that satisfies the aforementioned conditions. Any of the available analyses of the mechanisms governing such restrictions is unproblematically compatible with the approach discussed here. ${ }^{10}$

Sentences containing proper names are represented in DRT by means of conditions containing what is commonly called an 'anchor'; for instance, the sentence 'Mary is running' is represented as

$$
\left[<_{x}, \text { Mary }>\right][x \text { is running }]
$$

where the anchor $<_{x}$, Mary $>$ constrains the assignment of values to $x$ to Mary herself. Thus, this structure is evaluated as true iff there is a value for $x$ identical to Mary and it is running, i.e., iff Mary is running. In this apparatus, sentences containing indexical expressions may also be interpreted (with respect to a given context $c$ ) by means of representations involving an anchor. For instance, in virtue of the character associated with 'I', straightforward uses (in $c$ ) of 'I' are responsible for an anchor $<_{x}$, $\mathbf{i}>$, where $\mathbf{i}$ is $c_{i}$, the individual occupying the agent co-ordinate of $c$ (in

${ }^{9}$ I simplify considerably, and I present the result in a typographically more tractable format than is customary in the presentation of DRT.

${ }^{10}$ For recent discussions of this issue see, among many others, Recanati 1996 and Stanley and Szabo 2000. 
the symbolism from section three, $\mathbf{i}$ is the unique element in $C_{i}$ ). So, typical uses of 'I am running' may be interpreted by means of the representation

$$
\left[<_{x}, \mathbf{i}>\right][x \text { is running], }
$$

and are thus true with respect to a context $c$ as long as the agent of $c$ is running.

Turning now to generics, consider for instance the sentence

(5) a wolf takes a mate for life.

Normally, such a sentence is employed so as to convey a quasi-universal content, to the effect that typical wolves take a mate for life. Following a standard account (see Heim 1988, 190-195), it seems reasonable to suppose that this reading is derived from the interpretation of a hidden 'generic' operator, i.e., that the input for semantic evaluation is, roughly, of the form 'GEN a wolf takes a mate for life'. Within DRT, the operator 'GEN' is interpreted as giving raise to a representation of the form

$[x$ is a wolf $] * \rightarrow[x$ takes a mate for life $]$

(see Kamp and Reyle 1993). This representation is in turn evaluated as true iff every normal assignment of values to $x$ that verifies the condition on the left hand side of the starred arrow also verifies the other condition, that is, iff any typical wolf takes a mate for life.

Let us then return to the cases with which this essay is concerned, namely non-specific uses of, for instance,

(1) you get in trouble with that move

(2) do not put off until tomorrow what you can do today.

The readings for these sentences that are of interest here do not intuitively express a singular content, but rather convey a message of a general import: generally, people get in trouble with the indicated move, and typical days are unsuitable for procrastination. As these paraphrases indicate, it seems at least prima facie plausible to hypothesize that these readings are obtained on the basis of mechanisms parallel to those for the generic interpretation of, say, (5). If this hypothesis is taken seriously, it 
follows that (1) and (2) are appropriately analyzed by appealing to a (hidden) generic operator, that is, roughly, as in

(1*) GEN you get in trouble with that move

and

(2*) GEN do not put off until tomorrow what you can do today.

According to the proposal sketched above, these inputs are in turn interpreted (with respect to a context $c$ ) by means of the structures

$(1 * *)[x, \mathbf{a}] * \rightarrow[x$ gets in trouble with that move $]$

and

$(2 * *)[x, \mathbf{t}] * \rightarrow$ [you should not put off until the day following $x$ what you can do on $x$,

that is, they are assigned the truth-conditions that, respectively, every typical assignment to $x$ such that $x=\mathbf{a}$ gets in trouble with that move, and every typical assignment to $x$ such that $x=\mathbf{t}$ is unsuitable for procrastination to the following day — where $\mathbf{a}$ and $\mathbf{t}$ are the appropriate contextual co-ordinates. ${ }^{11}$

The suggestion that cases such as (1) or (2) be interpreted by appealing to the generic operator yields interesting results when conjoined with the hypothesis from section two, pertaining to the contexts appropriate for the interpretation of non-specific uses of indexicals. As I argued there, the contexts in question are not only distinct from the contexts of utterance, they are also gappy contexts: no particular individual is supplied as the addressee relevant for the evaluation of 'you' in (1), and no particular time is singled out as the semantic interpretation for 'today' and 'tomorrow' in (2). It follows that the foregoing analysis of these sentences with respect to

${ }^{11}$ More precisely, the clause for (2) should read: 'do not put off until the day following the 24 hour period including $\mathbf{t}$...', or something along these lines. For simplicity's sake I also ignore the indexical 'you' (in all likelihood to be analyzed impersonally), and I disregard the possible complications related to the fact that (2) is not a declarative sentence. 
contexts of this type involves what may be called empty anchors, that is, anchors that fail to restrict the interpretation of the variable they contain. More formally, given the notion of a context $c$ presented in section two, that is, $c=\left\langle C_{i}, C_{a}, C_{t}\right\rangle$, an indexical such as 'you' may now be deemed responsible for an anchor $<_{x}, C_{a}>$. An assignment $f$ of values to $x$ is compatible with an anchor of this type iff either (i) $C_{a}=\{\mathbf{a}\}$ and $f(x)=\mathbf{a}$, or (ii) $C_{a}$ $=\varnothing$. Similarly, mutatis mutandis, for other indexicals and the respective coordinates. Thus, the representation $(1 * *)$, when interpreted with respect to a context gappy in its addressee co-ordinate, is associated with the truthconditions that any typical individual within the relevant domain of individuals gets in trouble with a certain move. By the same token, when $(2 * *)$ is interpreted with respect to a context gappy in its temporal co-ordinate, the resulting truth-conditions require that, given any typical time, what can be done on that day should not be postponed to the following day. As explained above, and as with any other example independently of the peculiarities of non-specific indexicals, which domain happens to be relevant, and what qualifies as 'typical' within that domain, are questions that need to be assessed vis á vis a variety of background assumptions appropriate for the given conversational setting.

A few comments are in order before I conclude, pertaining to the employment of (1) or (2) in conversational exchanges. According to the theory I am advocating, sentences such as these may be semantically associated with a generic content, to the effect that, for instance, normal players tend to get in trouble with a certain move. This result is obtainable from straightforward semantic mechanisms, that is, from the customary characters for 'you' or 'today' together with the resources for the interpretation of generics. Still, the desired outcome is obtained only if these mechanisms are accompanied by a 'contextual shift' such that the appropriate parameters are distinct from certain co-ordinates of utterance. From the point of view of the theory of communication, as I remarked when discussing the simpler examples from section two, cases of this kind are particularly demanding: successful communication may take place only if one's audience indulges in the required shift, and at least temporarily disregards the parameters which, in the majority of normal situations, would correctly be taken as relevant. This fact is in turn responsible for the kind of 'limited productivity' that seems to affect the use of non-specific indexicals: although in the appropriate setting many indexicals may successfully be employed according to the pattern I dis- 
cussed in this essay, the examples the majority of speakers would suggest are relatively standardized sentences, such as 'you just can't tell', or proverbial statements such as 'you can lead a horse to water but you can't make it drink', 'better look now than seek anon', or (2).

It would be a mistake to interpret this evidence as supporting the conclusion that these examples are to be interpreted idiomatically: obviously non-idiomatic cases such as (1) or the use of 'if I have a dog, I take good care of it' in section two are correctly interpretable by any intelligent speaker competent in her use of the first and second person pronouns. ${ }^{12}$ But it would also be incorrect to suppose that, once a semantic regularity is at work of the kind I discussed, any indexicals may always be employed successfully so as to communicate a content of a general import. To the contrary, it is an important outcome of my analysis that, from the point of view of the efficiency of communication, particularly demanding mechanisms are at work in instances such as the impersonal use of 'you': one's audience needs to realize that the correct reading is to be derived from the assumption of a highly unusual, 'gappy' context of interpretation. It is thus to be expected that successful employment of the semantic regularities I unveiled may need to be accompanied by conventional practices: most (though, importantly, not all) of the examples I discussed are relatively standardized, and to some extent encoded within the standard vocabulary of competent users of the language in question.

\section{Conclusion}

My analysis of (1) or (2) is grounded on two at least prima facie reasonable hypotheses: that such examples bear interesting similarities to generic statements, and that the parameters in the context of utterance do not provide the correct interpretations of the indexicals. The resulting picture yields the intuitively desired readings, and remains immune from the shortcomings of the alternatives I criticized in section one. Unlike an account in terms of lexical ambiguity, it provides an explanation of the systematic mechanisms responsible for the non-specific uses of indexical expressions, and is thus clearly compatible with the fact that such uses are available in other languages, and that distinct indexicals are uniformly

${ }^{12}$ For the suggestion of an idiomatic analysis of some examples, see Everett 2002. 
evaluated in the desired fashion. Moreover, unlike Nunberg's proposal, my proposal yields a complete explanation of the required semantic result, and provides the needed quasi-general interpretation regardless of the properties actually instantiated by the items within the context of utterance.

In the view I proposed, the semantic profile of an indexical, that is, its conventional semantic meaning (character) is as sanctioned by the classic approaches to indexical languages — with the exception of the minor adjustment needed to take care of the hitherto unexplored possibility of gappy contexts. For instance, in my view, 'you' is associated with a character such that, for any context $c=\left\langle\ldots C_{a} \ldots\right\rangle$, its semantic value is the unique individual in $C_{a}$, that is, the context's addressee, if any such individual exists. In accordance with this character, straightforward uses of such an expression are responsible for semantically encoding singular contents, of the type discussed within the literature in the 'direct reference' tradition. That, in the presence of particular contextual-shifts, and under the influence of appropriate operators, general propositions may occasionally emerge, is thus a conclusion hardly incompatible with the fundamental lesson of the approach that tradition defended.

Stefano Predelli

Department of Philosophy, University of Nottingham

University Park, Nottingham NG7 2RD

stefano.predelli@nottingham.ac.uk

\section{References}

Corazza, E., W. Fish, and J. Gorvett. 2002. Who Is I? Philosophical Studies 107: 121.

Everett, A. 2002. Predelli on Procrastination. Analysis 62: 160-6.

Heim, I. 1988. The Semantics of Definite and Indefinite Noun Phrases. New York: Garland Publishing, Inc.

Kamp, H., and U. Ryle. 1993. From Discourse to Logic. Introduction to Modeltheoretic Semantics of Natural Languages, Formal Logic and Discourse Representation Theory, Part 1. Dordrecht: Kluwer Academic Publishers.

Kaplan, D. 1977. Demonstratives. In Joseph Almog, John Perry, and Howard Wettstein (eds.), Themes From Kaplan. Oxford University Press, 1989. 
Lewis, D. 1980. Index, Context, and Content. In S. Kanger and S. Öhman (eds.), Philosophy and Grammar, Reidel. Reprinted in Papers in Philosophical Logic, Cambridge University Press, 1998.

Nunberg, G. 1993. Indexicality and Deixis. Linguistics and Philosophy 16: 1-43.

Predelli, S. 1998. Utterance, Interpretation, and the Logic of Indexicals. Mind and Language 13: 400-414.

Recanati, F. 1996: Domains of discourse. Linguistics and Philosophy 19: 445-475.

Recanati, F. 1993. Direct Reference. From Language to Thought. Oxford: Blackwell Publishers Ltd.

Richard, M.: 1990, Propositional Attitudes. An Essay on Thoughts and How We Ascribe Them, Cambridge University Press, Cambridge.

Romdenh-Romluc, K. 2002. Now the French are invading England! Analysis 62: 34-41.

Smith, Q. 1989. The Multiple Uses of Indexicals. Synthese 78: 167-91.

Stanley, J., and Z. G. Szabó. 2000. On Quantifier Domain Restriction. Mind and Language 15: 216-261. 